\title{
Ultrastructral Analysis of Synapse in a Drosophila of the Swedish Mutation Which Causes Early-Onset Alzheimer's Disease Using Focused Ion Beam Microscopy
}

\author{
Se Jin Park ${ }^{1}$, Andreas Schertel ${ }^{2}$, Sung Sik Han ${ }^{1} *$ \\ ${ }^{1}$ Laboratory of cell engineering and 3D structure, School of Life Sciences and Biotechnology, Korea \\ University, Seoul, Korea \\ ${ }^{2}$ Carl Zeiss NTS GmbH, Oberkochen, Germany \\ *Corresponding author: sshan@korea.ac.kr
}

In brain research, analysis of neural structure and synaptic connectivity between neurons is often used to identify the cause of symptoms and precisely characterize neurodegenerative diseases. Indeed, disease brains, understanding how the neural microcircuitry affects the function of the nervous system is the principal goal[1]. Therefore, defining the structure of the neural circuitry and synaptic connections between neuron will be significant issues in forthcoming brain research.

Among the neurodegenerative brain diseases, Alzheimer's disease (AD) is one of the most progressive disease that leading cause of dementia. Brain of $\mathrm{AD}$ patients indicate two major pathognomonic protein deposite: Extracellular amyloid plaque (AP) which are generated by amyloid precursor protein (APP). And intracellular neurofibrillary tangle (NFT) which composed of and brain-specific microtubule binding protein tau. In AD brain, highly neurotoxic forms of accumulated $\mathrm{A} \beta$ cause synaptic dysfunction, memory defects, functional and structural changes at the synapse and ultimately dementia [2]. Therefore, it is necessary the structural study of brain as a basis for understanding psychiatric disorder and their treatments.

To further advance understand the structure of $\mathrm{AD}$ brain, we analyzed the three-dimensional (3D) architecture of the presynaptic terminal using Focused ion beam (FIB). FIB are among the most useful tool for the 3D reconstruction. In a FIB machine, automated cycle of milling and imaging produce a series of image similar in appearance to TEM serial sections [3],[4].

In this study, we analyzed structural change within a Mushroom body (MB) which plays a important role for learning and memory in insects. The mushroom body is a central control neuropil that receives multimodal input. we used transgenic line of Drosophila melanogaster Swedish mutant APP (Swe-APP). Swe-APP cause early onset Alzheimer's disease by increase production of A $\beta$. In this study, we suggest the altered characteristic feature in Alzheimer's disease model. 
Reference

[1] Defelipe J. Front Neurosci (2008) 10

[2] Iijima-Ando K et al. PLoS One.(2009) 8310

[3] Knott G et al. J. neuroscience. (2008) 2959

[4] Drobne D et al. Microscopy research and technique (2007) 895
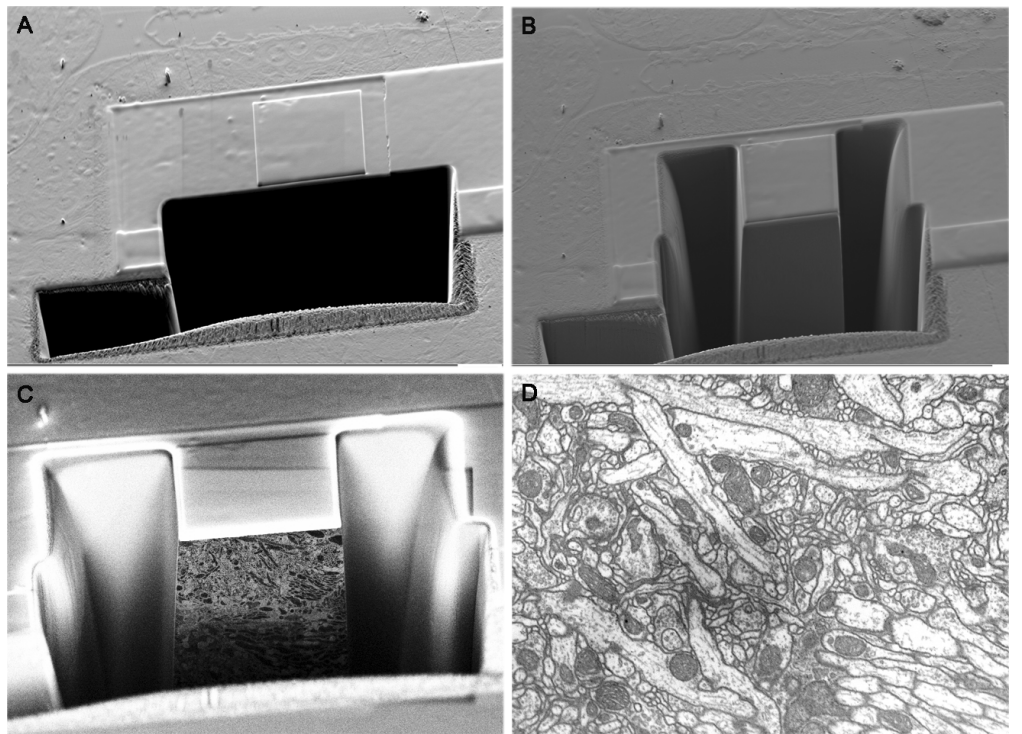

Fig 1. Images of FIB processing steps
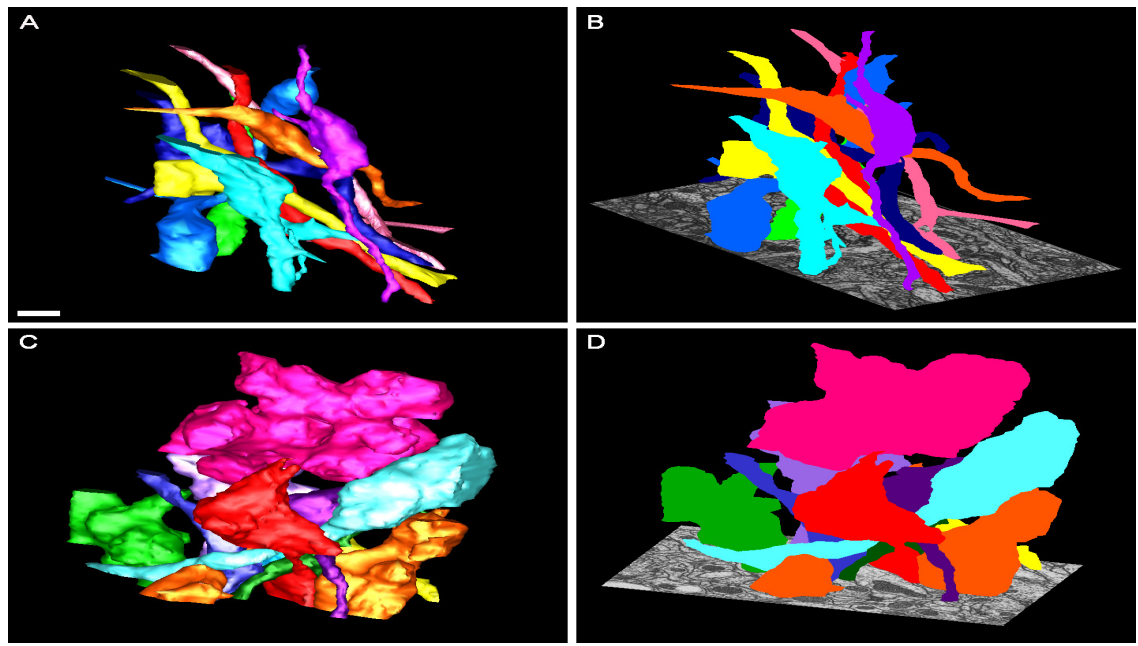

Fig 2. 3D reconstruction of presynaptic axons in Normal and AD 